\title{
Explaining temporal qualia
}

\section{Matt Farr ${ }^{1}$ iD}

Received: 4 March 2019 / Accepted: 26 September 2019/Published online: 9 January 2020

(C) The Author(s) 2020

\begin{abstract}
Experiences of motion and change are widely taken to have a 'flow-like' quality. Call this 'temporal qualia'. Temporal qualia are commonly thought to be central to the question of whether time objectively passes: (1) passage realists take temporal passage to be necessary in order for us to have the temporal qualia we do; (2) passage antirealists typically concede that time appears to pass, as though our temporal qualia falsely represent time as passing. I reject both claims and make the case that passage-talk plays no useful explanatory role with respect to temporal qualia, but rather obfuscates what the philosophical problem of temporal qualia is. I offer a 'reductionist' account of temporal qualia that makes no reference to the concept of passage and argue that it is well motivated by empirical studies in motion perception.
\end{abstract}

Keywords Time $\cdot$ Temporal passage Temporal experience $\cdot$ Motion perception Cognitive illusions

\section{Introduction}

There is a supposed 'flow-like' element of our experience of things like motion and change that is commonly understood as an appearance or representation of time passing. To point to some token examples from the literature: Norton (2010 p. 24) holds that 'the passage of time is one of our most powerful experiences'; Davies (1996, p. 275) takes the 'sensation of a flowing time' to be 'basic to my experience of the world'; and Le Poidevin (2007, p. 76) suggests that 'we just see time passing in front of us, in [...] any motion or change at all'. The idea of the passage or flow of time being given in experience is widespread but rarely expressed in terms that go beyond

Matt Farr

mail@mattfarr.co.uk

1 Department of History and Philosophy of Science \& Trinity College, University of Cambridge, Cambridge, UK 
suggestive metaphors. Nonetheless, it is common even for antirealists about the passage of time- those who deny that the passage of time is a fundamental feature of the world above and beyond the temporal aspects of our experience-to nonetheless concede that time appears to pass, such that our temporal experience constitutes an illusion in this respect. Call this idea 'illusionism'. Much contemporary literature in philosophy of time is devoted to providing a positive account of illusionism by explaining how and why time appears to pass. This has involved a recent focus on appeals to cognitive science, particularly the various empirical studies of motion perception, to explain why time appears to pass. ${ }^{1}$

This project is based on a mistake. There are two independently legitimate philosophical problems here that if run together serve to create basic confusions concerning each: (1) the ontological question of whether time really passes, independently of our experience; and (2) how to explain the phenomenal character of our temporal experience, particularly our perception of things like change and motion, in light of the relevant research in cognitive science (time perception illusions, psychophysics experiments, fMRI scans, etc.). Call (1) the problem of temporal passage, and (2) the problem of temporal qualia. There is no doubt that there is something it is like to perceive movement and change, and this is ubiquitously compared to the idea of time itself flowing like a river or passing by like an object. The central argument of this paper is that not only is such a concept of temporal passage not needed to explain temporal qualia, but that it is wholly irrelevant to temporal experience in general and has actively distorted both what the problem of temporal qualia has been taken to be, and also what kind of a philosophical problem the debate about temporal passage poses. The contention of the paper is normative: the problems of temporal passage and temporal qualia should be kept separate; and temporal qualia should be explained in passage-free terms.

The paper is structured as follows. Section 2 sets the terms for the paper by separating the problem of temporal passage from the problem of temporal qualia. Section 3 sets out what temporal qualia are, and draws an analogy between the relationship between temporal qualia and passage and the mind-body problem. Section 4 outlines four alternative explanatory models for temporal qualia, arguing that passage-realist models fail to offer any explanatory advantage with regard to our temporal qualia. In Section 5, I argue that time perception illusions should not be read as supporting the claim that temporal qualia are illusory, but rather motivate a 'reductionist' account of temporal qualia according to which temporal qualia are in general reliable indicators of change and motion in world. Section 6 sets out reductionism about temporal qualia in further detail, defending it on naturalist grounds by demonstrating that it is both continuous with and supported by empirical studies in time perception: first, reductionism offers a pluralist account of temporal qualia as consisting of a cluster of loosely related cognitive responses to physical change and motion, rather than offering a unified representation of passage, in-keeping with the pluralism of mechanisms involved in time perception; second, reductionism is supported by the phenomena of

\footnotetext{
${ }^{1}$ See Paul (2010), Norton (2010), Prosser (2012, 2013, 2016), Hoerl (2014), Phillips (2014), and Baron et al. (2015).
} 
motion blindness, where subjects lack certain kinds of motion qualia. Section 7 is the conclusion.

\section{Temporal passage and temporal qualia: two independent philosophical problems}

\subsection{Passage realism}

Temporal experience is often offered as evidence in favour of the 'A theory of time', and against the ' $\mathrm{B}$ theory of time'. The $\mathrm{A}$ and $\mathrm{B}$ theories of time are standardly taken to disagree over the ontological status of temporal passage. The A theory holds that the following two facts hold independently of our existence and our temporal location: (1) there is a privileged 'present' moment of time; (2) time passes. The B theory rejects both (1) and (2), holding that any beliefs we have about the specialness of the present, or of the objectivity of the flow of time, are to be explained as features of our temporal perspective, rather than perspective-independent features of the world. ${ }^{2}$ Though (2) is often understood in terms of the movement of the present moment, the concept of temporal passage is conceptually distinct from (1). 'Temporal passage' and 'time flow' are at base heuristic metaphors: we know what it is like to see a river flow or an object pass us by, and the idea behind passage realism is that our temporal experience as a whole is like this in that time itself appears to pass or flow. Such a metaphor is clearly problematic, since there appears nothing intrinsically special about the flow of a river of the passing-by of an object that the perception of which would constitute a perception or awareness of time as passing any more than any other kind of motion or change. But such details about how to explicate the concept of passage and its alleged phenomenological features can be put to one side since my central aim is to get rid of the metaphor in the explanation of temporal qualia.

I will instead reserve the term 'temporal passage' in this paper for the idea of something over-and-above the B-theoretic features of the world that has some important connection to the kinds of processes and experiences we ordinarily term 'temporal'. To unpack this a bit: the B theory holds the world to contain a number of temporal facts which are expressible in terms of relative spatial and temporal locations, such as that there are temporally-extended objects that take on different properties and positions at different times. Passage realism holds that beyond the Bfacts, time has some intrinsic dynamic quality that serves to distinguish it from space, which is a feature of the world and not merely part of our conscious representation of it. Since I am interested only in temporal passage, and not the idea of a privileged

\footnotetext{
${ }^{2}$ Though the B theory is antirealist about passage, it standardly assumes a preferred direction of time, as implied by its usage of 'earlier than' as an ordering relation. I elsewhere (Farr 2012, 2018; Farr, The C theory of time, unpublished) introduce and defend an alternative ' $\mathrm{C}$ theory' of time, according to which time neither passes nor has a privileged direction. The $\mathrm{C}$ theory, I contend, treats temporal qualia in the reductionist way I defend in this paper.

${ }^{3}$ We can imagine someone denying that time has a privileged present moment whilst still holding time to pass in a sense in which space does not-for example, see Maudlin (2007, ch. 4).
} 
present moment, I will use the terms 'passage realism' and 'passage antirealism' to refer to the two sides of the debate rather than 'A theory' and 'B theory'. 4

\subsection{Passage antirealism}

Passage realism has been subject to a number of famous critiques: McTaggart (1908) argues that the concept of temporal passage is incoherent; Broad (1938) and others ${ }^{5}$ object that temporal passage requires the existence of multiple time dimensions; Eddington (1928) argues that the concept of temporal passage plays no role in physical theory; Putnam (1967) and Rietdijk (1966) hold that temporal passage is incompatible with special relativity theory; and Gödel (1949) argues that temporal passage is inconsistent with general relativity theory. A consequence of this lopsided dialectic is that passage antirealism has had a relatively free ride, being explicated primarily in terms of what it rejects. However, the passage antirealist nonetheless requires a positive account of why time has the peculiar experiential features that it does. A popular move is to regard passage as a kind of illusion associated with our temporal experience that requires input from cognitive science - for instance (Paul 2010) argues that change and motion illusions provide an important insight into how we could come to have an illusion of temporal passage.

\subsection{Eliminating passage-talk}

My contention, which I detail in Sections 5 and 6, is that insofar as empirical psychology bears on the problem of temporal qualia, it serves to favour a reductionist account of temporal qualia whereby they are understood as phenomenal features of our range of abilities to detect real or illusory motion and change. I make the case that the analysis of human motion and change perception provides no empiricallymotivated grounds for holding either that (a) our temporal qualia ontologically or causally depend upon the passage of time, or (b) our temporal qualia form a unified representation of a mind-independent phenomenon of temporal passage that accords to the passage realist's concept of passage. As such, there is no need to draw a connection between the problems of temporal passage and temporal qualia, and thus reductionism offers a more promising approach. Reductionism is an account of how to understand what temporal qualia are, rather than an account of how to understand temporal passage. Reductionism is most naturally understood as a passage antirealist position, since it holds that there is no interesting link between our experience and the concept of temporal passage, undermining the central claims of the passage realist accounts I will consider. There is nonetheless logical space for someone to be a reductionist about temporal qualia whilst holding that time passes for some independent reason. This is a consequence of treating the problems of temporal qualia and temporal passage as wholly independent of each other.

\footnotetext{
${ }^{4}$ Indeed, the passage realism and A/B theory debates themselves come apart in various ways. For instance, Maudlin (2007) defends passage realism without the A theory, and Tallant (2010) defends a version of the A theory without passage realism.

${ }^{5}$ This objection to temporal passage has been raised by Dunne (1939), Smart (1949) and Black (1959).
} 
Siblings of such a reductionist position about temporal phenomenology have been outlined $^{6}$ and defended ${ }^{7}$ in the recent literature on the grounds that the claim that temporal phenomenology is systematically illusory is conceptually problematic and/or unintelligible. In particular Hoerl (2014) suggests that it is a mistake to hold that we have a phenomenology of temporal passage at all. This is a claim about the phenomenological character of temporal experience; indeed, Hoerl (p. 192) notes that this is a further error theory to that of the illusionist: the illusionist's belief that we have non-veridical passage phenomenology is itself an illusion. While such a position is welcome, I will not argue that we do not have passage phenomenology, nor that our phenomenology in itself is what should motivate a passage-antirealist attitude over a passage-realist one. On the contrary, I take it that we do have qualia that are worth grouping and characterising as 'temporal', and these have a phenomenal character that is commonly associated with the idea of time as passing. However insofar as we do have temporal qualia, I argue that it is a mistake to regard such qualia as functioning as a representation of time as passing, since such a position is not forced through philosophical argument, nor is such a claim useful in categorising the ways in which we can successfully or unsuccessfully represent things like motion or change. Rather, my central claim is that very tendency to relate the problems of temporal passage and temporal qualia in the first place serves only to confuse two independent philosophical problems: nothing is gained in the explanation of temporal qualia by referring to the concept of temporal passage.

\section{What is the problem of temporal qualia?}

A quale is a phenomenal, qualitative aspect of an experience, and qualia is the plural form. The qualia of some experience are generally described as the 'what-it's-like' properties of having that experience, such as what it is like to experience the specific shades of turquoise of the sea around the Great Barrier Reef. As such, qualia are necessarily of a first-person nature; there are no qualia without experiencers. In the temporal case, experiences of change and motion are widely appealed to as having distinctively temporal qualia, with some holding these to be to some degree experiences of the passage of time, such as Le Poidevin's claim that we 'see' the passage of time in 'any motion or change at all.' Visual perception of change and motion are the most typically cited instances of the passage-like features of our experience, and we'll focus primarily on these. ${ }^{8}$

\footnotetext{
${ }^{6}$ Baron et al. (2015) term a related position 'veridicalism', which holds both that time does not pass, and that we do not experience time as passing, hence temporal phenomenology is largely veridical and not systematically illusory. Miller et al. (2018) detail a range of different veridicalist positions.

${ }^{7}$ Deng (2013) and Hoerl (2014) each express scepticism as to whether we have a phenomenology of passage, and argue that the passage-antirealist (for Deng, the 'B theorist'; for Hoerl, the 'reductionist' about temporal passage) possess sufficient resources to account for the apparently animated aspects of change and motion perception without recourse to the passage realist's ontology.

${ }^{8}$ The examples of change and motion we consider in this paper concern only visual perception. There are various other sensory modalities with which we experience time, such as sound and touch, that we will not consider. See Phillips (2014) for a recent survey piece that covers cross-modal temporal experience.
} 


\section{1 'Temporal qualia' as an umbrella term}

We can group change and motion qualia and other related kinds of qualia ${ }^{9}$ - i.e. the phenomenal character associated with veridical or illusory perception of change and motion-under the general label 'temporal qualia'. In this sense, 'temporal qualia' can be considered an umbrella term that refers to any qualia we typically associate with the concept of time. I shall use the term in this neutral way, to refer to temporal qualia as a whole and not to imply that there is some unique phenomenological property shared by temporal qualia that other, non-temporal, types of qualia lack. ${ }^{10}$ The following facts are uncontroversial: first, things change and move in the world; second, we experience things as changing and moving; third, our experience of change and motion has particular phenomenological features that we typically associate with the concept of temporal passage; fourth, we can and do have change and motion qualia that do not correspond to actual change or motion in the world, owing to perceptual illusions.

To fix on the example of motion for a moment: there is something it is like to see motion, and we standardly have motion qualia in response to seeing a moving body, such as a bird flying past the window. Moreover, it is important that this set of qualia is not simply a perception of motion, since perceptual illusions can trigger non-veridical motion qualia in the absence of motion, and conversely cases of motion blindness (which we consider in Section 6) show that one can have awareness of motion without corresponding motion qualia. ${ }^{11}$ What is less clear and more contentious is whether any part of our experience corresponds to temporal passage in an analogous way.

\subsection{Are temporal qualia representational?}

Though the term 'qualia' is used to denote a variety of different concepts in the philosophical literature on perception, I shall use it to refer to the phenomenal character of experience in general. ${ }^{12}$ The central debate regarding qualia is whether they are representational in nature, with 'qualia' sometimes being used to denote only non-representational features of experience. The distinctive 'flow-like' or 'animated'

\footnotetext{
${ }^{9}$ It is often noted that there is something it is like to see one event succeed another, and to perceive different lengths of duration, and as such succession and duration arguably involve relevant temporal qualia. I will only refer to motion and change qualia for sake of brevity, but this is not to imply that these are the only kinds of temporal qualia.

${ }^{10}$ See Prosser (2013 pp. 80-82) for a useful discussion of a related issue.

${ }^{11}$ Throughout this paper, I use 'motion' to refer to the relative motion of an object with respect to an observer.

${ }^{12}$ Tye (2018) notes there are at least three popular alternative usages of the term: (2) in the context of sense data theory, referring to the non-representational qualitative aspects of sense data; (3) as nonrepresentational features of experience in general (independent of the wider claims of sense data theory); and (4) as intrinsic, ineffable aspects of experience.
} 
features of temporal qualia are commonly implied in the philosophy of time literature to be representational in nature: passage realists standardly take their position to be motivated by experience in that time appears to us as passing and we should take physical time to possess such a property; and illusionists, though they are antirealist about temporal passage, take our temporal qualia to constitute an illusion insofar as they amount to an inaccurate representation of time as passing.

The reductionist account of temporal qualia I defend does not take temporal qualia to be representational in this sense, and as such is in accord with the popular view of qualia in general as non-representational. However, reductionism is very specific in this regard. First, there is no 'passage quale'-i.e. some qualia purely associated with temporal passage that accompanies motion and change qualia. Second, to pick on a specific type of temporal qualia, we should at best think motion qualia to function as a generally reliable indicator of real motion, but not to indicate anything further, such as there being an intrinsically flow-like quality of moving things. As a result, temporal qualia do not in any sense form a representation of time as passing.

\subsection{Temporal qualia and the explanatory gap}

Just as the mind-body dualist takes physicalism to be unable to account for qualia due to an explanatory gap between the mental and physical, ${ }^{13}$ the passage realist can hold a passage-antirealist ontology (i.e. the B-facts) to be insufficient to account for temporal qualia. Following Chalmers' (1996) famous 'zombie' argument against physicalism, the passage realist may take it to be possible for a world to share the same B-facts as our world but lack the passage of time, and for inhabitants of such a world to hence lack temporal qualia, such that their experience is somehow deficient to ours in key respects in that things don't appear to 'flow' in the same way. ${ }^{14}$ Maudlin (2007), for instance, implies that in a passageless world, people would not be conscious - we come back to this issue in Section 4. Passage is often taken to be the 'something extra' required to bridge the gap and connect the manifest and scientific images of time: Eddington (1928, p. 34; my emphases) notes that '[s] omething [i.e. passage] must be added to the geometrical conceptions comprised in Minkowski's world before it becomes a complete picture of the world as we know it;' and Davies (1996, p. 275; my emphasis) stresses that the 'sensation of a flowing time [...] is an aspect of time of great significance that we have so far overlooked in our description of the physical universe'.

It is not necessary to hold that there is such an explanatory gap. To extend the analogy between the philosophy of time and philosophy of mind, we can think of the passage antirealist as akin to the physicalist: just as the physicalist takes qualia to necessarily follow from the physical facts (e.g. pain qualia is just what it's like for something/anything to experience pain), the passage antirealist can take temporal

\footnotetext{
${ }^{13}$ Cf. Levine (1983).

${ }^{14}$ Though passage realism is in this sense analogous to kinds of dualism (such as Chalmers' property dualism), it doesn't follow that the passage realist must reject physicalism. The passage realist could be a physicalist but simply hold that B-facts are not sufficient to account for the properties of physical time.
} 
qualia as a necessary consequence of beings like us interacting with a B-theoretic ontology; temporal qualia don't need any extra explanans. ${ }^{15}$

This problem is famously addressed by Williams (1951), who holds that it is a mistake for passage realists to think that "they alone are "taking time seriously", (p. 458). As Williams notes, this falsely assumes that the passage antirealist does not wish to account for the temporal idiosyncrasies of experience. On the contrary the passage antirealist simply holds that the apparently dynamic aspects of temporal experience that are generally termed as 'passage' or 'flow' are fully entailed by their picture of time. This point is further emphasised by Prosser (2013), noting that since 'both [passage realist and antirealist] theories, on their own terms, predict the same experiences $[, \ldots]$ experience does not favour one theory over the other' (p. 71). As such, we should not hold that 'our experience of tense and temporal becoming [...] overwhelms any B-theoretic arguments against the reality of tense' as Craig (2000, p. 165 ) claims, nor that 'realism about tense is uniquely capable of making sense of the phenomenology of temporal experience' as Hare (2010, p. 762) suggests on behalf of the passage realist. Sentiments such as these compromise the dialectic of the passage debate by unfairly stacking the deck against the passage antirealist through implying that they do not take their theory to entail nor take seriously temporal qualia.

It follows that the passage antirealist can simply deny the explanatory gap and hold instead that temporal qualia are perfectly possible without temporal passage; the antirealist is not seeking to deny that we have temporal qualia but rather offering a theory of it that does not depend upon passage. What this brings to the fore is that the dispute between passage realism and antirealism is not whether temporal qualia are to be explained, but rather what is considered necessary in order for them to be explained. The passage realist holds the passage of time to play crucial role in explaining how it is that we have temporal qualia, and so by their lights the passage antirealist lacks an explanation of how it is that we have temporal qualia. The passage antirealist rejects this, holding instead that no explanation is needed for how we can come to have temporal qualia in the absence of passage. It follows that the problem of temporal passage is a priori in nature, and not, therefore, something to be settled with reference to temporal qualia.

\subsection{What is the explanatory problem of temporal qualia?}

In treating the problem of temporal qualia independently of the problem of temporal passage, I have two aims. Firstly, temporal qualia does not equate to an experience of passage. In this context, Paul (2010) coins the phrase "experience as of" in order to speak of our passage-like phenomenology without implying that it is veridical (i.e. corresponds to real passage). However, my second aim is to go a step further and avoid prejudging that our experience of time involves experience 'as of' passage

\footnotetext{
${ }^{15}$ Perry (2001) adopts this analogy in the case of 'nowness'. Perry argues that Mellor's (1998) argument for the B theory amounts to a temporal version of Jackson's (1986) 'knowledge argument', with A-theorists holding that a property of 'nowness' is required to explain temporally-indexical beliefs.
} 
at all. ${ }^{16}$ Rather, the very idea that temporal qualia in any coherent sense represent time as passing — whether veridically or erroneously — is a mistake that leads to misrepresenting what it is about temporal qualia that stands in need of explanation.

Consider two alternative ways to pose the explanatory problem of temporal qualia:

Q1. Why does it appear to us as though time passes?

Q2. How do we account for the specific kinds of temporal qualia we have?

In the next two sections, I argue that Q1 poses a problem that does not stand in need of explanation. In Section 4 I argue that the claim that passage is necessary for temporal qualia plays no role in accounting for what is special about motion and change qualia. In Section $5 \mathrm{I}$ argue that passage antirealists, insofar as they do not share this claim, are not required to address Q1-on their terms, the question is a non-issue. As such, the claim that temporal qualia are illusory as regards temporal passage, and related attempts to explain away passage as being a cognitive illusion, are fundamentally misguided. Q2, on the contrary, represents a tractable and interesting issue that is far more amenable to input from cognitive science. Sections 5 and 6 detail specific ways in which cognitive science bears upon Q2, and crucially, how such empirical investigation fits best with a reductionist account of temporal qualia.

\section{How (not) to explain temporal qualia}

In separating the concepts of temporal qualia and temporal passage, we can ask more detailed questions about how they relate. First, is temporal passage necessary for temporal qualia? And secondly, do temporal qualia represent temporal passage? These two issues are independent but are often run together, and considering the different combinations of yes/no answers to these questions gives us a range of positions as laid out in Table 1. I'll first summarise these positions before critiquing them.

Representational Realism. Temporal passage is necessary for temporal qualia. Temporal qualia represent temporal passage.

Representational realism corresponds to the idea that we directly experience temporal passage. On this account, our temporal qualia track temporal passage insofar as they are a veridical conscious representation of temporal passage and are caused by temporal passage.

Inferential Realism. Temporal passage is necessary for temporal qualia. Temporal qualia need not represent temporal passage.

Inferential realism relaxes the idea that we have direct experience of temporal passage, instead holding temporal passage to be a necessary precondition for temporal qualia, and hence that we can legitimately infer that such a thing must exist in order to account for our having temporal qualia.

\footnotetext{
${ }^{16}$ See Deng (2013) and Hoerl (2014) for criticisms of the claim that we have experiences 'as of' passage.
} 
Table 1 The different strategies for explaining temporal qualia

\begin{tabular}{lllll}
\hline & RR & IR & Illusionism & Reductionism \\
\hline Is TP necessary for TQ? & $\checkmark$ & $\checkmark$ & $x$ & $x$ \\
Do TQ represent TP? & $\checkmark$ & $x$ & $\checkmark$ & $x$ \\
\hline
\end{tabular}

$\mathrm{TP}=$ Temporal passage; $\mathrm{TQ}=$ temporal qualia; $\mathrm{RR}=$ representational realism; $\mathrm{IR}=$ inferential realism

Illusionism. Temporal passage is not necessary for temporal qualia. Temporal qualia represent temporal passage.

Illusionism holds our experience of time to be fundamentally misleading in that although time appears to us as passing, this is a cognitive illusion and fails to correspond to a mind-independent feature of the world.

Reductionism. Temporal passage is not necessary for temporal qualia. Temporal qualia do not represent temporal passage.

Finally, reductionism holds that temporal qualia neither depend upon nor represent temporal passage: change and motion qualia (and any other kind of temporal qualia) are simply indicators of things changing or moving over time. When I see a lorikeet flying past my window, any flow-like qualities of my motion qualia are simply what it is like to represent the lorikeet's motion as it takes on a series of different positions in my visual field relative to the background scenery over a short period of time.

\subsection{Representational realism}

Though I have argued in Section 3 that the problem of temporal passage is a priori in nature and so is not to be settled by reference to temporal qualia, it is worth going through independent shortcomings of passage-realist approaches to the problem of temporal qualia. First, representational realism is the most extreme position of the four approaches to temporal qualia in that temporal qualia function as the direct perception of temporal passage. Though this view is reasonably intuitive, it is subject to a basic epistemic problem that calls for a more sophisticated explanatory model of temporal qualia. Eddington (1928, p. 91) notes that such a view 'is tantamount to an admission that consciousness, looking out through a private door, can learn by direct insight an underlying character of the world which physical measurements do not betray'. If temporal passage is something over and above the existence of changing and moving things in the world, then the problem is how we could we gain epistemic access to such a thing. Temporal passage is not something present in physics textbooks; we don't need to know any special properties of the passage of time to account for the behaviour of any known phenomena (except allegedly our first-person experience of time). As such, this requires some kind of special connection between our brains and temporal passage that 'goes around' the physical sciences. There is good reason to suppose then that if passage is to play a role in the explanation of temporal 
qualia, it is not in the sense that our temporal qualia are offering a glimpse into an underlying feature of the world.

\subsection{Inferential realism}

Inferential realism shares with representational realism the idea that temporal passage plays a key role in explaining temporal qualia, but it relaxes the claim that we experience temporal passage-instead we can think of temporal passage as something that is inferred by the realist from the general 'feel' of temporal qualia. Inferential realism instead holds that the relationship between passage and temporal qualia is that the former is necessary for the latter; without temporal passage, we wouldn't have temporal qualia. Recent advocates of such a position include Maudlin (2007) and Norton (2010). ${ }^{17}$ Maudlin considers what we can call temporal zombie worlds ${ }^{18}$ — worlds physically identical to our own but stipulated to not contain temporal passage - and argues that 'unless one already has begged the central question' against passage realism, it follows that 'the state of this [zombie] world is so unlike the physical state of anything in our universe that to suppose that there are mental states at all is completely unfounded' (Maudlin 2007, p. 124). In a similar vein, Norton argues that temporal passage is required to explain why our psychological timeline matches the temporal order of events in the world, holding that 'if passage is an illusion, there must be some mechanism that blocks us perceiving the future' (Norton 2010, p. 30), implying like Maudlin that without passage we would not have the kind of temporal experience that we do.

Though inferential realism avoids the epistemic problem faced by representational realism, it faces a more basic problem regarding the relationship between temporal passage and temporal qualia: there is no sense in which inferential realism can explain why the paradigm instances of temporal qualia — motion and change qualia—are special vis-à-vis passage. Take Maudlin's suggestion that inhabitants of a passageless temporal zombie world would not have mental states: if temporal qualia and temporal passage are related in this way, then any conceivable mental state would stand in the same dependence relation to temporal passage regardless of whether it concerns experience of time, or thoughts about the size of elephants' ears, or whatever. This account fails because it is too general: there is no unique link between temporal passage and the characteristically temporal kinds of qualia, such as motion and change qualia, meaning that such a dependence relation between temporal qualia and temporal passage provides no explanation of what makes temporal qualia special for the

\footnotetext{
${ }^{17}$ It should be noted that both Maudlin and Norton make claims that sound as though temporal passage is in some sense experienced, more in line with representational realism. However, both put forward models in which the key relation between temporal qualia and temporal passage is that the former counterfactually depend upon the latter, in line with inferential realism.

${ }^{18}$ Maudlin's points are made specifically against Price's (1996) version of Williams' (1951) thought experiment, involving a world physically identical to our own but stipulated to not contain temporal passage. Price argues that there is no reason to suppose beings in such a world would experience time differently from ourselves, since on any fair mapping of physical states from the passage world to the no-passage world, the brain states of individuals would be mapped from the former to the latter.
} 
passage realist. Prosser (2013, p. 82) picks on this generality of explanation in passage realist accounts of temporal qualia, noting that even if such accounts were true, temporal qualia would fail to constitute a 'unique, perception-constituting relation to the passage of time'. ${ }^{19}$ But this point goes deeper: even if the realist is happy to weaken the perceptual claim and accept that temporal qualia function as something less than a perception or representation of passage, as inferential realist does, the key explanatory problem remains: there is no account at all of why motion and change qualia in particular should be analogous in any sense to the passage of time, since other mental states equally dependent on temporal passage clearly are not. As such, inferential realism does not explain temporal qualia.

\section{Abusing your illusion}

Putting passage realist models to one side, it is common to portray passage-antirealist positions (i.e. B-theories) as holding some kind of illusionism about temporal qualia. For instance, from the passage-antirealism camp: Smart (1980, p. 3) holds that 'the alleged passage of time [...] is an illusion'; and Prosser (2012, p. 92) holds that 'the B-theory cannot be regarded as fully satisfactory until an adequate account has been given of the illusion of passage'. Moreover, passage realists typically cite the perceived flaws of illusionism as supporting their position: Davies (1996, p. 275) is 'repelled by the claim that [passage] is only an illusion or misperception'; Maudlin (2007, p. 135) holds that 'all the philosophizing in the world will not convince us that these facts [that time appears to pass] are mere illusions'; and Norton (2010, p. 23) remarks that '[e]xplaining passage away as an illusion is an instance of a desperate stratagem that has been used to ill effect elsewhere when we become too eager to explain away an awkward fact'.

I share the latter sentiments that it is undesirable to hold temporal qualia to be systematically illusory, but think that rather than supporting passage realism, it supports a best-of-both-worlds option, which is to reject the idea that temporal qualia represent passage in the first place. The study of time perception better supports the claim that temporal qualia are in general veridical, with time perception illusions highlighting specific exceptional circumstances in which temporal qualia erroneously indicate change and motion in unchanging and motionless objects.

\subsection{When temporal qualia are illusory}

There is a range of well-studied time perception illusions that are instructive with respect to temporal qualia since they constitute cases where temporal qualia demonstrably fail to function as accurate representations of the world. These are illusions that involve subjects experiencing change and motion qualia in the absence of changing or moving stimuli. These include (but are not restricted to):

\footnotetext{
${ }^{19}$ Prosser specifically talks of temporal phenomenology rather than qualia, but for our purposes this difference in terminology makes no odds.
} 
Phi Phenomenon. A series of still images of differently positioned dots presented to an observer in rapid succession give rise to the illusory perception of a single enduring object in continuous motion between the dot locations (cf. Wertheimer 1912; for a demonstration, see Bach 2014a).

Motion Aftereffect. A subject's visual system adapts to a moving image, such that after the stimulus is removed, the subject appears to perceive the inverse motion (cf. Mather et al. 1998; for a demonstration, see Bach 2014b).

Peripheral Drift. A still image appears to contain motion as the eye scans around the image (cf. Fraser and Wilcox 1979. A particularly vivid example of this is Kitaoka's rotating snakes illusion [Fig. 1]).

Each of these three kinds of motion illusion demonstrates a different way in which temporal qualia can be illusory. The two that are of most interest are the motion aftereffect and peripheral drift illusions. In the case of motion aftereffect illusions, temporal qualia are illusory insofar as they continue after a motion-involving stimulus is removed. A paradigm example is the 'waterfall illusion', where after staring at a waterfall for some time and then shifting focus to the adjacent rock face, the rock face appears to be moving upwards, in the opposite direction to the water. Motion aftereffect illusions are the product of our visual system adapting to a continuous motion by counteracting to the motion, with the result of reducing the intensity of the perceived motion and the corresponding sense of disorientation. A consequence of this is that once the stimulus is removed, the neural adaptation creates the illusion of motion in the opposite direction to that of the original stimulus. Thus, one has motion qualia that are an effect of real motion, but continue in the absence of that motion, and also give the appearance of motion in the opposite direction. The resulting motion qualia are not veridical, although they are a consequence of past motion. Peripheral

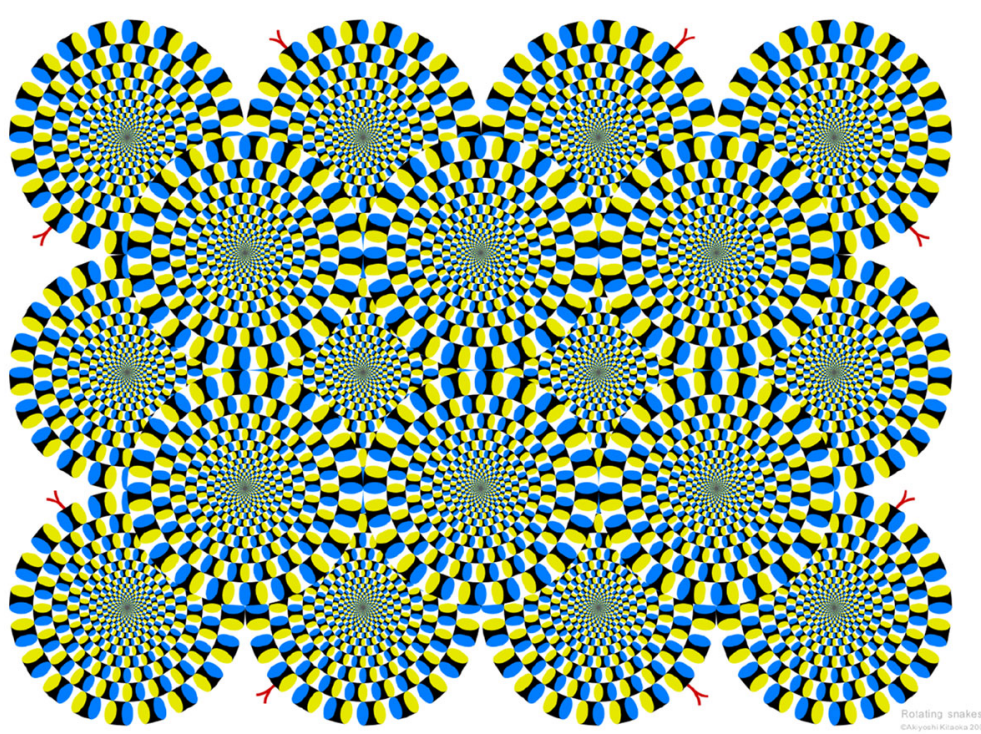

Fig. 1 The Rotating Snakes illusion (Kitaoka 2016) 
drift illusions are of greater interest in this regard insofar as the motion qualia are not a consequence of actual motion in the object being perceived. As the name suggests, a peripheral drift illusion involves the illusion of motion in the periphery. Such illusions incorporate repeated patterns of asymmetric luminance that in peripheral vision appear as motion in the dark-to-light direction, as vividly depicted in Fig. $1 .{ }^{20}$ The motion qualia are generated without the presence of a moving stimulus, unlike motion aftereffect illusions. ${ }^{21}$

The phi phenomenon represents a different kind of illusion with respect to temporal qualia. In the paradigm case of a pair of flashing dots sequentially appearing at opposite ends of a screen, the observer typically projects a single dot moving at high speed from side to side. ${ }^{22}$ Such a case is interesting with regard to temporal qualia insofar as it provides an example in which the perceived motion of the dot is illusory. Moreover, this case has been taken as an instance of human subjects using an 'endurantist' representation of the dots as a single, enduring dot (for instance, both Paul 2010 and Prosser 2013 argue along these lines. I consider Paul's treatment of this later in this section).

Time perception illusions demonstrate that temporal qualia can be produced in the absence of actual physical change or motion in the relevant object being observed, and hence are illusory in this respect. A consequence of this is that there is a key sense in which temporal qualia are, in general, veridical: our temporal qualia, except under specific well-studied and well-understood conditions, reliably indicate and are triggered by change and/or motion of the perceived objects. Time perception illusions represent anomalous cases, and the study of the psychophysical and physiological details of these aids the understanding of how the brain produces the appearance of motion and change, and under precisely what conditions these fail to be veridical.

\subsection{Why illusions don't imply illusionism}

The veridical nature of temporal qualia in ordinary conditions stands in direct contrast to the central claim of illusionism, which is that temporal qualia are systematically illusory insofar as they represent something - temporal passage - that does not exist. Paul (2010) makes the case that time perception illusions, particularly the phi phenomenon, are illustrative in understanding illusionism. In the case of the phi phenomenon-let's again use the case of the flashing dots-, the brain fills in the space between the two dot locations so it appears as though a single dot is traveling at high speed between the two locations. Paul suggests by analogy:

\footnotetext{
${ }^{20}$ See Fraser and Wilcox (1979) for the original presentation of the peripheral drift illusion and Faubert and Herbert (1999) for analysis. Backus and Oruç (2005) additionally offer an analysis of Kitaoka's rotating snakes illusion.

${ }^{21}$ Backus and Oruç (2005) argue that this type of illusion is due not to the shift of one's visual focus around the image, but rather 'fast and slow changes over time in the neuronal representation of contrast $[\ldots]$ or luminance' (p. 1055).

${ }^{22}$ In the case of the 'color-phi' illusion, the dots are of different colours, and so the illusory moving dot gradually changes colour.
} 
[W]hen we have an experience as of passage, we can interpret this as an experience that is the result of the brain producing a neural state that represents inputs from earlier and later temporal stages and simply 'fills in' the representation of motion or of changes. Thus, according to the [illusionist], ${ }^{23}$ there is no real flow or animation in changes that occur across time. Rather, a stage of one's brain creates the illusion of such flow, as the causal effect of prior stages on (this stage of) one's brain. (Paul 2010, p. 352; my emphasis)

This gives a specific picture of how an illusionist explanation of temporal qualia might work. The idea is that by drawing on the sense in which we can have motion qualia in the absence of corresponding motion in the relevant physical object, the passage antirealist can give a general heuristic for how in principle one can have temporal qualia in a world without passage. I've argued that this is not a task which the passage antirealist need take up. Instead, the reductionist about temporal qualia rejects the idea that we have 'experiences as of passage' insofar as (a) there is no single 'passage quale' — rather the 'flow' or 'animation' are features of temporal qualia in general (such as motion qualia) — , and (b) temporal qualia in general don't represent time as passing in the way in which the concept of temporal passage is ordinarily understood (as something over and above the B-facts). In light of this, the reductionist denies that there is an illusion to explain here: if the flow and animation are simply what it is like to perceive real or imaginary motion and/or change, then it is not illusory, since it in general reliably tracks real change and motion.

There are strong independent reasons for taking such an error theory to be in vain. Although there is certainly a general analogy between, on the one hand, our ability to project motion qualia in the absence of a moving stimulus, and on the other hand, the illusionist's claim that we project temporal passage onto reality as a whole despite there being no such thing, there is a major problem with such an approach: there are key senses in which the term 'illusion' is explanatorily useful in the first case that fail to carry over to the second and ultimately promote unnecessary confusion about the nature of our temporal experience. Take the rotating snakes illusion (Fig. 1): the illusion consists in the appearance of motion as a result of looking at a still image. Such illusions involve a perceptual stimulus giving rise to misleading qualia. A hallmark of such illusions is that there are independent ways of verifying that the stimulus lacks such a quality-in this case, we can independently verify in a number of ways that Fig. 1 is a still image, and so confirm that our motion qualia are misleading us. ${ }^{24}$ Indeed a key feature of perceptual illusions is that they are localised in that they generally do not apply wholesale across all of our sensory modalities, allowing us to establish that they are indeed illusions. However, the illusionist's account of the

\footnotetext{
${ }^{23}$ Paul uses the term 'reductionist', so it is necessary to distinguish the position to which she refers from the position I call reductionism. Paul is referring to the reductionist about temporal passage, such as the B theorist, who takes our experience of time to not depend upon the reality of temporal passage. This picks out passage antirealism in general. She also takes 'reductionism' to be committed to the idea that passage is an illusion. I use 'reductionism' as shorthand for 'reductionism about temporal qualia', which holds that temporal qualia reduces to motion qualia, change qualia, and the like.

${ }^{24}$ I use the rotating snakes illusion here as my example, but the same reasoning applies also to Paul's example of the phi phenomenon.
} 
illusion of temporal passage is of a different kind. ${ }^{25}$ In the case of illusionism, temporal qualia are both permanently and systematically illusory insofar as: (1) every temporal quale erroneously represents something — temporal passage - that does not correspond to anything in reality; (2) the illusion of passage is consistent across all of our sensory modalities.

With regard to (1), the illusionist is committed to the unwelcome consequence that ordinary experiences of motion and illusory motion qualia are equally illusory with respect to passage, and as such, there is a major disanalogy between standard cognitive illusions and the alleged illusion of passage: according to the illusionist, we are under the illusion of passage both in the case that we observe real motion, such as seeing a bird fly by, and in the case that we observe illusory motion, such as with the rotating snakes illusion. ${ }^{26}$

With regard to (2), the illusionist's alleged illusion of passage would presumably also have to be cross-modal in nature, affecting all of our sensory mechanisms, so that time would not only visibly appears to pass, but also sound and feel as though it were passing. There is no doubt that the rotating snakes illusion would be all the more powerful and interesting were it to also produce the illusion of auditory and tactile motion together with visual motion, but there is no such all-encompassing motion illusion to be found in the literature.

Whereas the concept of an illusion is useful and informative in the case of whether motion qualia tracks actual motion, it is by contrast unhelpful to use the same concept with respect to whether all types of temporal qualia track temporal passage. In the former case, we know how to measure whether something has moved in a way that corresponds to one's motion qualia (in part due to the fact that such illusions are typically restricted to one of the sensory modalities), but in the latter case, it is not clear what could possibly count as a measurement of whether or not time is passing. ${ }^{27}$ All in all, the alleged analogy between the illusionist account of passage and cases of change and motion illusions breaks down upon analysis. The usefulness and empirical meaning of the term 'illusion' in cases of motion and change illusions does not carry over to the case of temporal passage; nothing, it seems, is gained philosophically by taking an illusionist account of temporal qualia. As such, illusionism's key thesis fails to offer any explanatory advantage regarding temporal qualia over reductionism.

On the contrary, it is preferable to consider illusions like the rotating snakes (Fig. 1) in reductionist terms. When considering the illusory motion qualia, the reductionist seeks no further explanation as to why in addition to the motion qualia we

\footnotetext{
${ }^{25}$ As Norton (2010, p. 23)notes: '[w]e know what illusions are like and how to detect them. Passage exhibits no sign of being an illusion'.

${ }^{26}$ Alternatively, Deng (2013) suggests that the analogy drawn by Paul is itself ambiguous: either the illusion of motion in the phi phenomenon and the illusionist's alleged illusion of passage are distinct illusions; or they are different aspects of the same illusion. If the former, Deng suggests that 'it is hard to see what the second illusion [the illusion of passage] could be' (p. 377). If the latter, it is unclear what is to be gained by the analogy.

${ }^{27}$ Indeed, on standard passage-realist accounts, the passage of time is continuous and ubiquitous-it is not something that stops and starts or varies from place to place.
} 
appear also to experience animation or flow in the image. To think there is something further that stands in need of explanation is, according to reductionism, to make a category mistake - the appearance of animation or flow in the image is just what it is like to represent motion. In holding the 'dynamic' and 'flow-like' qualities of temporal experience to be features of what it is like to represent motion or change, the reductionist gives up the idea that something further than motion-i.e. temporal passage-is represented by temporal qualia.

\section{An appetite for reduction}

To recapitulate, reductionism about temporal qualia holds that:

(1) our temporal qualia amount to no more than the sum of change and motion qualia and related kinds of qualia;

(2) each of these are simply what it is like to perceive the relevant physical phenomenon (motion, change, etc.);

(3) there is no extra 'passage quale' that accompanies or unifies such qualia; and

(4) such qualia do not amount to a collective representation of temporal passage as something over and above a passage-antirealist (B-theoretic) ontology.

This section outlines in more detail the reductionist account of temporal qualia and shows how it is supported by and continuous with various empirical work in the study of time perception.

\subsection{Understanding reductionism}

The distinction between reductionism and illusionism is analogous to that between moral naturalism and moral error theory. Moral error theory shares with moral realism the idea that moral statements are truth-apt and that they refer to a realm of non-reductive moral facts, but holds that there are no such facts and so moral statements are systematically false. Moral naturalism holds that moral statements refer to contingent worldly matters of fact, and in this way avoids both the epistemic problems of moral realism and error theory's undesirable consequence that all of our moral talk is outright false. Analogously, I've argued that the central problem with illusionism is precisely that it shares with passage realism the claim that our temporal qualia represent temporal passage, which is something over and above motion and change facts, and since illusionism is a passage-antirealist position, it consequently holds that our temporal qualia are systematically in error. I've argued that this errortheoretic claim of illusionism plays no important explanatory role regarding temporal qualia, and the passage antirealist is better off giving up the claim that temporal qualia represent temporal passage. Just as moral naturalism allows for empirical import into ethical questions, reductionism about temporal qualia shifts the focus of enquiry about temporal qualia onto empirical issues concerning time perception. 


\subsection{Pluralism about temporal qualia}

Reductionism gives a pluralist account of temporal qualia in that it takes temporal qualia to consist of a number of different kinds of qualia that are not necessarily unified by some particular phenomenological property. This pluralist account of temporal qualia makes sense of how we represent change and motion and under what circumstances our motion and change qualia are misleading. Indeed, the kinds of experience that we take to be temporal are in general quite varied and distinct, whether it be seeing the change of a flickering picture, the motion of a bird, sensing that a situation has endured for too long, or having the more abstract feeling of disorientation at time having elapsed after waking from an unexpected nap. This pluralism of temporal qualia is demonstrated even within our main case study of motion perception, which is standardly categorised into first and second orders. First order motion perception involves basic motion sensors that are sensitive to spatiotemporal variations in luminance and colour. Second order motion perception involves more complex qualities such as texture and contrast. The first and second orders differ in a number of ways. For instance, in the case of motion aftereffect, first-order motion detectors are more sensitive to the adaptation to the stimulus, since their aftereffects last for twice the duration as those of second-order detectors (Mather 1991, p. 164), ${ }^{28}$ and also second order but not first order motion detection tails off markedly in peripheral vision (Pantle 1992). ${ }^{29}$

Though it is an open question how exactly first and second order motion peception relate, a wide range of studies on cognitive impairments in patients indicate that first and second order motion detection owe to different neural pathways and mechanisms (cf. Vaina and Cowey 1996; Nishida et al. 1997; Greenlee and Smith 1997; Vaina et al. 1998). For instance, Vaina and Soloviev (2004, p. 197) suggest that 'first-order motion is carried out by mechanisms along the dorsal pathway in the occipital lobe, while the second-order motion by mechanisms mostly along the ventral pathway'. Such studies support a pluralistic understanding of motion perception, suggesting that motion qualia owe to a range of distinct neural processes in response to a range of different kinds of stimuli. This supports the wider idea that temporal qualia are associated in a number of different ways with a range of distinct kinds of perception. This pluralist view of temporal qualia does not cohere to the passage realist view that temporal qualia are a response to a single phenomenon (passage), nor to the shared view of passage realists and illusionists that temporal qualia are a unified representation of a single phenomenon (passage).

\subsection{Motion blindness and temporal qualia}

The study of motion blindness, or 'akinetopsia', is of particular relevance to the understanding of temporal qualia. In such cases, patients have been noted to have

\footnotetext{
${ }^{28}$ See also (Ledgeway and Smith 1994) for an examination of the asymmetric durations of the motion aftereffect in the cases of first and second order motion.

${ }^{29}$ For a recent survey of first and second order motion detection, see Nishida (2011).
} 
'perceptual experience of a moving target as if the visual stimulus remained stationary but appeared at different successive positions' (Zihl et al. 1983, p. 314), ${ }^{30}$ and so in effect have awareness of change in position without accompanying motion qualia. Most notably, with respect to explaining the flow-like quality of temporal qualia, Zihl et al. (1983, p. 315) describe an affected patient's ability to pour coffee as compromised 'because the fluid appeared to be frozen, like a glacier'. Zihl et al's study concerns a patient, LM, with acute akinetopsia, due to bilaterally symmetric damage to the lateral temporo-occipital cortex, such that LM complained of 'a loss of movement vision in all three dimensions' (p. 315), restricting her ability to judge the speed of approaching trains, pour drinks without overfilling, and cross roads; for instance LM reported that 'people [are] suddenly here or there but I have not seen them moving'. Studies on LM revealed that her perception of motion in depth was 'completely abolished' (p. 334), with some preservation of motion perception in targets moving along the horizontal and vertical axes of LM's inner visual field, though with no such motion perception in the periphery. The motion blindness was restricted to visual motion, with LM responding normally to both tactile and acoustic motion.

Zihl et al. (1983) furthermore studied LM's motion perception with respect to two of the motion illusions described in Section 5, first the phi phenomenon, and second motion aftereffect. LM lacked the ability to perceive phi-phenomenon: when presented with several variations of the successive presentation of two differentlypositioned dots, ' $[u]$ nder no combination of conditions did the patient report apparent movement. She always reported two independent light spots,' in contrast to the control subject. Similarly, LM had significantly limited susceptibility to the motion aftereffect, in comparison to a control subject. In the case of a left-to-right-moving stimulus:

The patient reported some kind of a motion aftereffects in only 3 of the 10 trials. Its duration, however, was rather short (1.2, 1.4 and $2.1 \mathrm{~s})$ when compared with the normal subject tested under the same conditions (mean duration $6.7 \mathrm{~s} ; 5$ inspection periods). The patient described the motion after effect as an 'unrest' of the pattern, but never as movement. (p. 330)

These and other studies (cf. Zeki 1991) on patients with varying degrees of akinetopsia fit with the reductionist view of temporal qualia in a key way: the partial loss of the ability to detect motion is accompanied by a loss of temporal qualia. Indeed, the literal flow of a fluid is specifically reported to be viewed as 'frozen [...] like a glacier' in the case of LM. It is hard to think of a more exact analogy with the static vs dynamic pictures standardly put forward within the debate between passage-realist and passage-antirealist theories of time (i.e. the central disagreement between A- and B-theorists). In this sense, we can see that the akinetopsic lacks certain temporal qualia, and accordingly there is a reduced flow-like aspect of experience. The reductionist explanation here could not be simpler-the covariance of the flow-like quality of experience with the ability to represent motion is explained in terms of the former being a feature of the latter; for the reductionist the apparently animated quality of

\footnotetext{
${ }^{30}$ Zihl et al. (1983) are here referring to a patient studied by Pötzl and Redlich (1911).
} 
perceiving real or imaginary motion is nothing further than having motion qualia; it is simply what it is like to perceive motion. The fact that the akinetopsic has restricted temporal qualia compared to the control subject both in cases of real and illusory motion is explained, according to reductionism, by the fact that the akinetopsic has a restricted ability to represent motion. ${ }^{31}$

For the passage realist and the illusionist there is extra conceptual apparatus with which to understand the case of akinetopsia, since these positions allow us to distinguish between motion qualia - what it is like for something to appear in motion and passage qualia - what it is like for time to appear to pass. There are two broad options: either (1) the loss of motion qualia does not affect the akinetopsic's passage qualia; or (2) the akinetopsic has diminished passage qualia. Let's go through each of these options in turn. In case of (1), it is unappealing to hold that the akinetopsic's passage qualia is unaffected, since the missing qualia - the flow-like quality of motion - seems to be a prime candidate for what constitutes the feeling of temporal passage on these accounts. ${ }^{32}$ As such, the second option would appear to be the preferred one, that motion blindness involves a loss of passage qualia.

In the case of (2), we require some account of why it is that motion qualia and passage qualia covary in this way. It may very well be possible to extend one's account of realism or illusionism to accommodate this covariance of motion and passage qualia in a coherent and elegant way. However, such a move would be motivated by one's background commitment to realism or illusionism rather than being motivated by the phenomena. Unless there are good independent empirical grounds for thinking that passage qualia could come apart from the set of things like motion qualia, change qualia, etc., then there is no motivation to adopt such positions in order to account for phenomena like motion blindness. One such possibility would be if there were a phenomenon whereby a subject could be stimulated to experience qualia consistent with the flow-like qualities of temporal qualia, but without accompanying motion or change qualia. The problems here are, first, it is hard to know how such a thing could possibly be established, and second, if such a thing were established, it would fail to explain why perceptions of change and motion appear to be distinctively temporal, as opposed to other kinds of qualia, after all.

The reductionist needs no such gymnastics. My suggestion is that the flow-like aspect of motion qualia - that which is extremely vivid in Fig. 1-is both (a) a prime

\footnotetext{
${ }^{31} \mathrm{An}$ interesting related case to that of akinetopsia is that of so-called 'miracle cure' patients, such as the case of SB (Gregory and Wallace 1963), who regained sight having been effectively blind since early childhood. SB subsequently had great difficulty tracking an individual object through movement, or whilst seeing it from different angles, resulting in a restricted ability to perceive an individual object as in motion. See Kelly (2005) for a discussion of this in relation to akinetopsia.

${ }^{32}$ One might suppose that there is still useful logical space here for distinguishing motion qualia from the appearance of passage for the following reason: in order for a fluid to appear frozen, is has to do so as time passes. I don't agree. First, such cases of motion blindness are usually understood in terms of time appearing to be like a series of still snapshots; while none of these visibly endure, they can be assigned an apparent duration with reference to the patient's other sensory modalities, since things like auditory time perception are unaffected in cases of visual motion blindness. Second, B theorists of time have provided plentiful resources for talking about things like temporal extension and duration, such as the idea of something being frozen for some period of time, without making reference to the idea of time as passing.
} 
example of the qualities of temporal qualia that are standardly taken to relate to the concept of temporal passage, and (b) just what it is like to ordinarily perceive real or illusory motion. In LM's case, the inhibited motion perception leads to a loss of motion qualia in cases of observing moving bodies and in being presented with visual motion illusions. If we are to look to empirical work in order to analyse and understand temporal qualia, the study of akinetopsia seems to be of special relevance, and such studies neatly fit a reductionist account of temporal qualia, and alone provide no additional need to make reference to the concept of temporal passage.

\section{Summing up}

Our conclusions are twofold. First, there are two importantly distinct explanatory programs concerning temporal qualia-(a) how temporal qualia relate to the concept of temporal passage, and (b) how a different kinds of temporal qualia are to be explained in terms of perception and cognition-, of which I've argued only the latter need be addressed. Second, I've argued for a reductionist account of temporal qualia over the alternative approaches (representational realism; inferential realism; and illusionism). Reductionism, unlike the alternative three approaches, considers temporal qualia entirely independently of the concept of temporal passage. This is welcome for a number of key reasons: (i) no explanatory advantage is gained with respect to temporal qualia by referring to temporal passage; (ii) the question of whether temporal qualia are illusory with respect to passage is entirely independent of whether temporal qualia are illusory with respect to change, motion and other temporal features of the world, and running the two together creates grounds for confusion; (iii) the reductionist account of temporal qualia is continuous with and well supported by empirical studies in time perception.

These conclusions address several core issues in the philosophy of time. Our experience of time is widely taken to be central to the debate between A and B theorists insofar as it's taken weigh in on whether one should be realist about temporal passage. However, on the contrary we've seen that the relevant phenomenological aspects of our temporal experience-temporal qualia - cannot settle the passage realism debate. This is because the realism debate ultimately hangs on the background issue of whether temporal passage is necessary for temporal qualia, and this amounts to a normative debate over how temporal qualia ought to be explained. Furthermore, it is commonly argued that the explanatory task for the passage antirealist (or Btheorist) is to account our dynamic experience of time in a static universe. However, to understand the passage antirealist's task in this way is to give too much value to the terms of the passage debate, and to run together the problems of temporal passage and temporal qualia. We've seen that insofar as empirical work in the psychology of time can illuminate the issue of temporal qualia, it does so in terms of how the varied types of temporal qualia that we ordinarily have are grounded in our cognition of processes in the world. Questions of this type are not only amenable to empiricallyinformed philosophy, but a considerable amount of relevant empirical work has been carried out and it is known in great detail how different kinds of temporal qualia are projected onto our experience by parts of our brain in response to certain triggers, 
and what functions these have and why we might have developed them. Reflection on such studies, I've argued, supports a reductionist account of temporal qualia, suggesting that our temporal experience consists of a range of different kinds of perception that in ordinary conditions reliably indicate various kinds of physical processes in the world, rather than collectively tracking or representing an independent phenomenon of temporal passage.

Acknowledgments This paper dates back to an abandoned chapter of my $\mathrm{PhD}$ thesis, and has evolved through discussions with colleagues at the universities of Sydney, Queensland and Cambridge. I'm especially thankful to Alex Holcombe, Patrick Goodbourn, Natalja Deng, Pete Evans, Milena Ivanova, Adrian Bardon, Antony Eagle and two anonymous referees for helpful comments, and to audiences in Queensland, Munich, Cambridge, Bristol and the 4th Annual IAPT Conference in Gargnano for all kinds of unexpected reactions.

Open Access This article is distributed under the terms of the Creative Commons Attribution 4.0 International License (http://creativecommons.org/licenses/by/4.0/), which permits unrestricted use, distribution, and reproduction in any medium, provided you give appropriate credit to the original author(s) and the source, provide a link to the Creative Commons license, and indicate if changes were made.

\section{References}

Bach, M. (2014a). Colour phi phenomenon. http://www.michaelbach.de/ot/col-colorphi/.

Bach, M. (2014b). http://www.michaelbach.de/ot/mot-adapt/index.html.

Backus, B.T., \& Oruç, I. (2005). Illusory motion from change over time in the response to contrast and luminance. Journal of vision, 5(11), 10-10.

Baron, S., Cusbert, J., Farr, M., Kon, M., Miller, K. (2015). Temporal experience, temporal passage and the cognitive sciences. Philosophy Compass, 10(8), 560-571.

Black, M. (1959). The "direction" of time. Analysis, 19(3), 54-63.

Broad, C.D. (1938). Examination of McTaggart's philosophy Vol. II. Cambridge: Cambridge University Press.

Chalmers, D.J. (1996). The conscious mind: in search of a fundamental theory. Oxford: Oxford University Press.

Craig, W. (2000). The tensed theory of time: a critical examination. Dordrecht: Kluwer.

Davies, P. (1996). About time: Einstein's unfinished revolution. Simon and Schuster.

Deng, N. (2013). On explaining why time seems to pass. The Southern Journal of Philosophy, 51(3), $367-382$.

Dunne, J. (1939). An experiment with time, 5th edn. London: Faber and Faber Limited.

Eddington, A.S. (1928). The nature of the physical world. Cambridge: Cambridge University Press.

Farr, M. (2012). Towards a C theory of time: an appraisal of the physics and metaphysics of time direction. Ph.D. thesis. Bristol: University of Bristol.

Farr, M. (2018). Causation and time reversal. The British Journal for the Philosophy of Science.

Faubert, J., \& Herbert, A.M. (1999). The peripheral drift illusion: a motion illusion in the visual periphery. Perception, 28(5), 617-621.

Fraser, A., \& Wilcox, K.J. (1979). Perception of illusory movement. Nature, 281, 565-566.

Gödel, K. (1949). A remark about the relationship between relativity theory and idealistic philosophy. In Schilpp, P.A. (Ed.) Albert Einstein: philosopher-scientist, volume 7 of the library of living philosophers (pp. 557-562). Evanston: The Library of Living Philosophers, Inc.

Greenlee, M.W., \& Smith, A.T. (1997). Detection and discrimination of first-and second-order motion in patients with unilateral brain damage. Journal of Neuroscience, 17(2), 804-818.

Gregory, R., \& Wallace, J. (1963). Recovery from early blindness. Experimental Psychology Society Monograph, 2, 65-129.

Hare, C. (2010). Realism about tense and perspective. Philosophy Compass, 5(9), 760-769. 
Hoerl, C. (2014). Do we (seem to) perceive passage? Philosophical Explorations, 17(2), 188-202.

Jackson, F. (1986). What mary didn't know. The Journal of Philosophy, 83(5), 291-295.

Kelly, S.D. (2005). The puzzle of temporal experience. In Brook, \& Akins (Eds.) Cognition and the brain: the philosophy and neuroscience movement. Cambridge: Cambridge University Press.

Kitaoka, A. (2016). http://www.ritsumei.ac.jp/akitaoka/index-e.html.

Le Poidevin, R. (2007). The images of time: an essay on temporal representation, Oxford University Press, Oxford.

Ledgeway, T., \& Smith, A.T. (1994). The duration of the motion aftereffect following adaptation to firstorder and second-order motion. Perception, 23(10), 1211-1219. PMID: 7899037.

Levine, J. (1983). Materialism and qualia: the explanatory gap. Pacific Philosophical Quarterly, 64(4), 354-361.

Mather, G. (1991). First-order and second-order visual processes in the perception of motion and tilt. Vision Research, 31(1), 161-167.

Mather, G., Verstraten, F., Anstis, S. (1998). The motion aftereffect: a modern perspective, Mit Press.

Maudlin, T. (2007). The metaphysics within physics. Oxford: Oxford University Press.

McTaggart, J.M.E. (1908). The unreality of time. Mind, 17(68), 457-474.

Mellor, D.H. (1998). Real time II. Routledge.

Miller, K., Holcombe, A., Latham, A.J. (2018). Temporal phenomenology: phenomenological illusion versus cognitive error. Synthese.

Nishida, S. (2011). Advancement of motion psychophysics: review 2001-2010. Journal of Vision, 11(5), 11-11.

Nishida, S., Ledgeway, T., Edwards, M. (1997). Dual multiple-scale processing for motion in the human visual system. Vision Research, 37(19), 2685-2698.

Norton, J.D. (2010). Time really passes. Humana. Mente: Journal of Philosophical Studies, 13, $23-24$.

Pantle, A. (1992). Immobility of some second-order stimuli in human peripheral vision. Journal of the Optical Society of America. A, 9(6), 863-867.

Paul, L.A. (2010). Temporal experience. The Journal of Philosophy, 107(7), 333-359.

Perry, J. (2001). Time, consciousness and the knowledge argument. In Oaklander, L., \& Perry, J. (Eds.) (pp. 81-93): Springer.

Phillips, I. (2014). Experience of and in time. Philosophy Compass, 9(2), 131-144.

Pötzl, O., \& Redlich, E. (1911). Demonstration eines falles von bilateraler affektion beider occipitallappen. Wiener Klinische Wochenschrift, 24, 517-518.

Price, H. (1996). Time's arrow and Archimedes' point: new directions for the physics of time. Oxford: Oxford University Press.

Prosser, S. (2012). Why does time seem to pass? Philosophy and Phenomenological Research, 85(1), 92-116.

Prosser, S. (2013). Passage and perception. Noûs, 47(1), 69-84.

Prosser, S. (2016). Experiencing time. Oxford: Oxford University Press.

Putnam, H. (1967). Time and physical geometry. The Journal of Philosophy, 64(8), 240-247.

Rietdijk, C.W. (1966). A rigorous proof of determinism derived from the special theory of relativity. Philosophy of Science, 33(4), 341-344.

Smart, J.J.C. (1949). The river of time. Mind, 58(232), 483-494.

Smart, J.J.C. (1980). Time and becoming. In Van Inwagen, P. (Ed.) Time and cause: essays presented to Richard Taylor (pp. 3-15). Dordrecht: Reidel.

Tallant, J. (2010). A sketch of a presentist theory of passage. Erkenntnis, 73(1), 133-140.

Tye, M. (2018). Qualia. In Zalta, E.N. (Ed.) The Stanford encyclopedia of philosophy (Summer 2018 ed.) Metaphysics Research Lab: Stanford University.

Vaina, L.M., \& Cowey, A. (1996). Impairment of the perception of second order motion but not first order motion in a patient with unilateral focal brain damage. Proceedings of the Royal Society of London B: Biological Sciences, 263(1374), 1225-1232.

Vaina, L.M., \& Soloviev, S. (2004). First-order and second-order motion: neurological evidence for neuroanatomically distinct systems. In The roots of visual awareness: a festschrift in honour of Alan Cowey, volume 144 of progress in brain research (pp. 197-212): Elsevier.

Vaina, L., Makris, N., Kennedy, D., Cowey, A. (1998). The selective impairment of the perception of first-order motion by unilateral cortical brain damage. Visual Neuroscience, 15(2), 333-348.

Wertheimer, M. (1912). Experimentelle studien über sehen von bewegung. Z Psychol Physiol Sinnesorgane, 61, 161-265. 
Williams, D.C. (1951). The myth of passage. The Journal of Philosophy, 48(15), 457-472.

Zeki, S. (1991). Cerebral akinetopsia (visual motion blindness). Brain: A Journal of Neurology, 114(2), 811-824.

Zihl, J., Von Cramon, D., Mai, N. (1983). Selective disturbance of movement vision after bilateral brain damage. Brain: A Journal of Neurology, 106(2), 313-340.

Publisher's note Springer Nature remains neutral with regard to jurisdictional claims in published maps and institutional affiliations. 